\title{
All-Optical Logic Gates: Designs, Classification, and Comparison
}

\author{
Pallavi Singh, ${ }^{1}$ Devendra Kr. Tripathi, ${ }^{1}$ Shikha Jaiswal, ${ }^{2}$ and H. K. Dixit ${ }^{1}$ \\ ${ }^{1}$ Department of Electronics and Communication, University of Allahabad, Allahabad 211002, India \\ ${ }^{2}$ Department of Physics, S. D. (PG) College, Muzaffarnagar, India \\ Correspondence should be addressed to Pallavi Singh; singh.pallavi73@gmail.com
}

Received 20 September 2013; Revised 1 February 2014; Accepted 9 February 2014; Published 19 March 2014

Academic Editor: Zoran Ikonic

Copyright (C) 2014 Pallavi Singh et al. This is an open access article distributed under the Creative Commons Attribution License, which permits unrestricted use, distribution, and reproduction in any medium, provided the original work is properly cited.

\begin{abstract}
The paper reviews the current status and designs of all-optical gates. Various schemes with and without semiconductor optical amplifiers are discussed and compared. The optical gates are classified according to their design structures. It is divided into two major divisions that is, nonsemiconductor optical amplifier based gates and semiconductor optical amplifier based gates. In nonsemiconductor optical amplifier based gates, different schemes have been proposed to create non-linearity which is discussed. The semiconductor optical amplifier based gates of different design structures are discussed to show the probe pulse that is modulated in different ways to obtain results.
\end{abstract}

\section{Introduction}

Today the demand for high bandwidth has rapidly increased to obtain the speed limit of electronic devices. The general purpose of all-optical signal processing is still on the horizon. Nowadays, prototype of all-optical logic gates at high bitrate are coming out from the laboratories. The researches are going forward in this field to make it possible. However, in optical signal processing the digital gates have complicated and cumbersome electrooptic conversion. To make alloptical systems, it is necessary that entire components which are used in optical networks such as add-drop multiplexer, packet synchronization, clock recovery, address recognition, and signal regeneration, and so forth should be all-optical elements. To make the dream come true the basic requirement is optical gates. Gates are the key elements to realize all-optical functions. Thus, to realize digital gates into alloptical logic gates at the same platform, it is necessary to develop several basic designs. It is impractical to design an optical component with some gates using ultrafast nonlinear interferometer (UNI), some gates with SOA and some with high nonlinear fiber (HNLF). So the design is only successful when all the gates are implemented with same technique. Alloptical logic gates are core logic unit to implement various alloptical systems for optical signal processing. To design optical gates it is necessary to implement a nonlinear medium which modulates the signal to produce the desired results. The nonlinearity may be generated in numerous ways such as using nonlinear loop mirror, nonlinear fiber, photonic crystal, filter, waveguide, thyristor, acoustic waves, or semiconductor optical amplifier. Therefore, there are many researches going on to realize all-optical signal processing systems which are already discussed in various papers. It may be classified in multiple ways according to the design structures. All-optical gates are divided in two basic structures as in Figure 1 which are without SOA and with SOA.

\section{All-Optical Gates}

All-optical gates may be constructed using the nonlinearity effect which is introduced without SOA or with SOA. Numerous ways of all-optical gates without SOA using length of the fiber, waveguide, circulator, filters, acoustic-optic waves, and changing the refractive index of the optical waveguide have been discussed in the first part. Gates constructed with SOA are discussed in the second part.

2.1. All-Optical Gates without SOA. The nonlinearity in silica fiber arises from the nonlinear index of refraction. The change in nonlinear refractive index gives rise to an intensity dependent phase of the optical field. The effect of nonlinear interaction between two copropagating signals in the fiber 


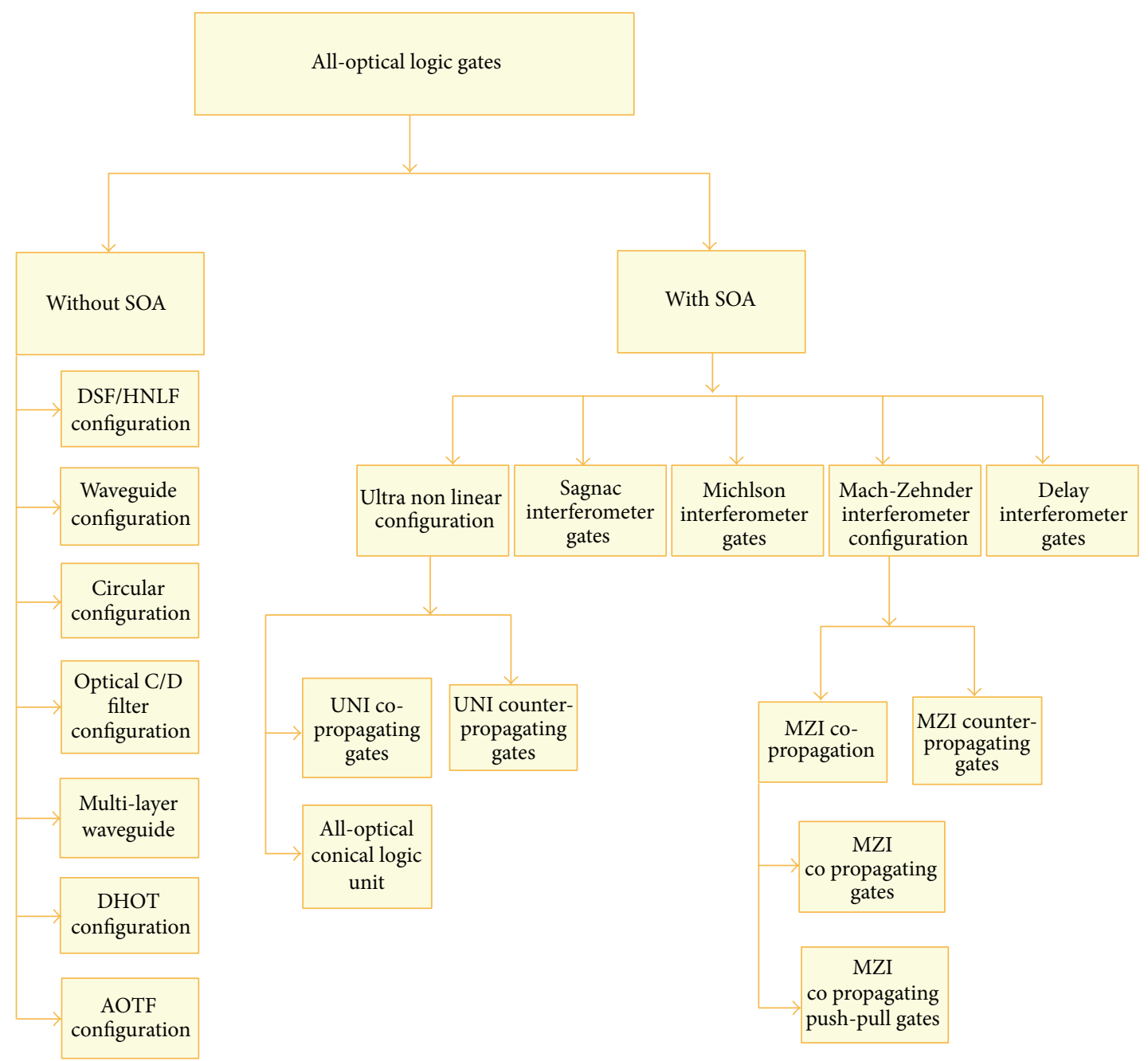

FIgURE 1: Classification of all-optical logic gates.

can be expressed by the change in electric field on one of the signals caused by the other after propagating through some distance in fiber. The intensity dependent refractive index of silica medium gives rise to three effects, self-phase modulation (SPM), cross gain modulation (XGM), and four wave mixing (FWM).

Self-phase modulation (SPM) occurs when intensity modulated signal travels through an optical fiber. The peak of the pulse travels slower than the wings. Due to this the wavelength of a pulse is stretched at the leading edge of the pulse and compressed on the trailing edge. Therefore, the trailing edge acquires a "blue shift" and leading edge acquires a "red shift." This modulates the signal and leads to broadening of the pulse.

Cross gain modulation (XGM) is another way in which intensity fluctuations affect the phase of a signal. Chromatic dispersion plays a significant role in gain modulation of the signal. Thus, the intensity fluctuation in the signal power of one channel propagating in the fiber modulates the phase of the other channel.

Four wave mixing (FWM) is a third-order nonlinearity and analogous to intermodulation distortion in the optical system. It is produced when beating between two channels at different frequencies modulates the signal phase at that frequency, generating new tones as side bands. The power of the side bands is always less than the signal power [1-3].

\subsubsection{Dispersion Shifted Fiber/High Nonlinear Fiber} (DSF/HNLF). The first design of Figure 2 consists of length of a fiber which introduces nonlinearity in the propagating signal. The length of the fiber introduced in the designs is of three types, dispersion shifted fiber (DSF), high nonlinear fiber dispersion shifted fiber (HNLF-DSF), and high nonlinear fiber (HNLF). The first design of Figure 2(a) introduced DSF that adds a constant shift due to the selfphase modulation (SPM) between the counterpropagating data at the output [4-6]. The counterpropagating data produces a cross phase modulation (XPM) which changes a pump power level and produces a constructive and destructive interference at the output. Both the data of same wavelength is used to avoid the four wave mixing (FWM).

In case of HNLF-DSF (Figure 2(b)), the data is depleted through XGM affected with the power transferred to the newly generated FWM component. When only one of the 


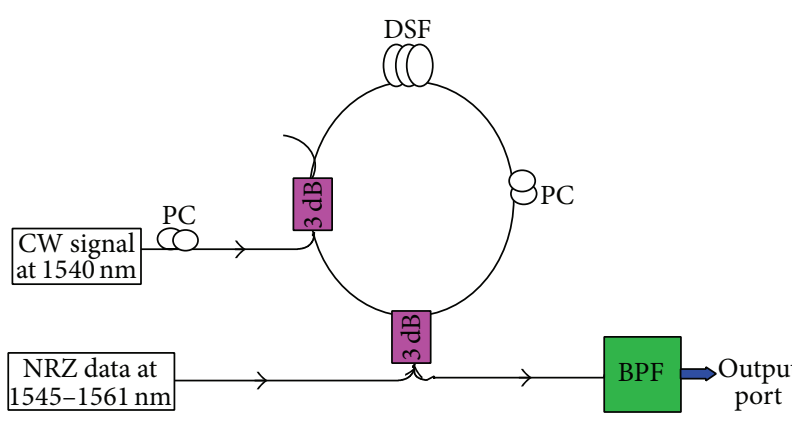

(a)

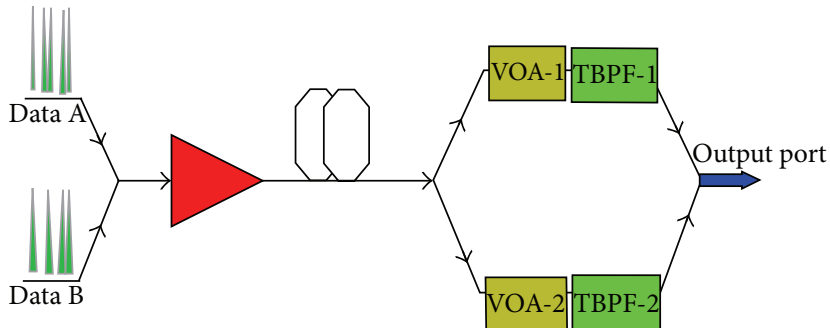

(b)

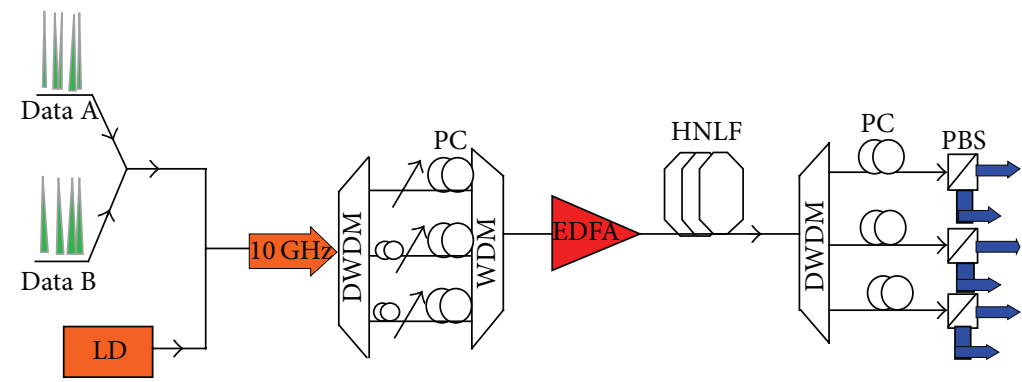

(c)

Figure 2: Design of gates consisting of (a) dispersion shifted fiber (DSF), (b) high nonlinear fiber-dispersion shifted fiber (HNLF-DSF), and (c) high nonlinear fiber (HNLF).

signals is present and launched into the HNL-DSF, it appears at the output, but if both the signals are present, no significant power appears at the output due to the state of polarization between the given data. By coupling these two output signals, one can achieve the desired results depending on the strength of the XGM $[7,8]$.

In the design (Figure 2(c)), all-optical logic gates are realized on the nonlinear polarization rotation (NPR) in HNLF. The polarization of light depends upon the intensity and relative polarization of the signal. When pump and probe signals travel through the HNLF, it introduces a nonlinear phase shift due to the SPM and XPM. The polarization of a probe signal changes and different gates can be realized [9-12]. This type of gate design using the length of fiber to produce a phase shift makes the design bigger and bad competitor.

2.1.2. Waveguide Configuration. In the design of Figure 3(a) two data $\mathrm{A}$ and $\mathrm{B}$ of different wavelengths are generated through microelectronic and mechanical system (MEMS) external cavity tunable laser. Both the data are coupled through mirror into the Fabry Perot chip (FP-chip). FP-chip basically has multimode wavelength output. As data passes through the cavity the signal experiences the nonlinearity effect. Due to the nonlinearity of the signals FP-chip is optically locked. The band pass filter (BPF) selects the wavelength which results as a gate operation [13].

The design consists of lights of different wavelengths injecting into the Si-wire waveguide with different peak power as in Figure 3(b). While travelling through the waveguide they experience two photon absorption which gives rise to the cross gain modulation. By adjusting the proper power of the pump and probe pulse we can get the results [14].

In Figure 3(c) two phase encoded data streams of different wavelengths are generated by two clock wave lasers and modulated with $33 \%$ duty cycle to produce RZ data. Both the data are copropagated with a continuous wave and fed into the chalcogenide $\mathrm{As}_{2} \mathrm{~s}_{3}(\mathrm{ChG})$ waveguide. The $\mathrm{ChG}$ waveguide offers broadband and flexible wavelength operation with ultrafast nonlinear response due to the kerr nonlinear index coefficient [15]. Due to a nondegenerate FWM process new wavelength is generated which is fed into a tunable band pass filter (TBPF) to extract the gate output.

The design without pump is used in periodically poled lithium niobate (PPLN) waveguide to produce gate output [16-18]. Here the waveguide is used to produce sum frequency generation (SFG) depending on the guide length. Two data are injected into the waveguide in which SFG occurs under the quasi-phase matching condition. When both of the data are the same the signal is depleted during the generation of sum frequency wave and finally output will be zero. If any one of the data is high, only one data will be depleted, and simultaneously another will still exist at the output.

2.1.3. Circulator. In Figure 4, two data of different wavelengths are generated which are passed through first $2 \times$ 2 coupler and second $2 \times 2$ coupler. Now both pump and probe are passed through Fabry Perot laser diode (FP-LD). The unique property of FP-LD is that if a single mode beam with slightly higher wavelength of longitudinal mode of FP$\mathrm{LD}$ is injected, the beam will experience the gain while the other modes of FP-LD are suppressed. When second beam 


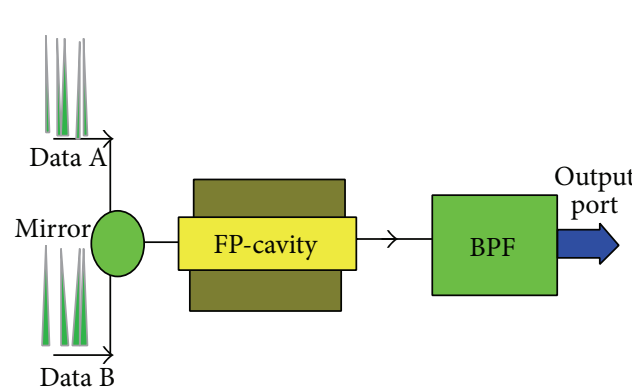

(a)

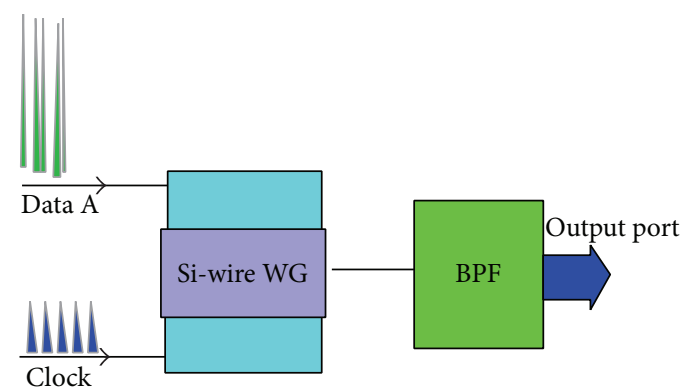

(b)

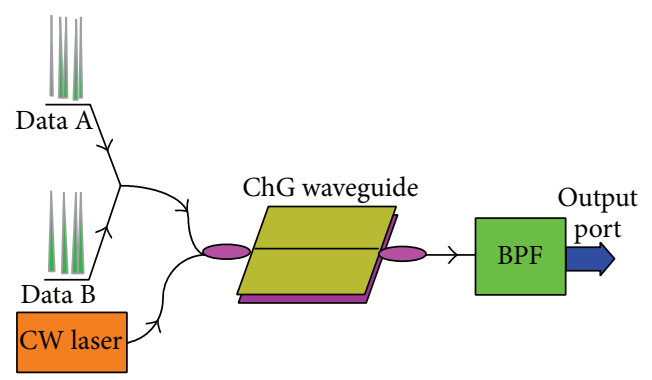

(c)

FIGURE 3: Design of gates consisting of (a) FP-cavity, (b) Si-wire, and (c) ChG/PPLN waveguide.

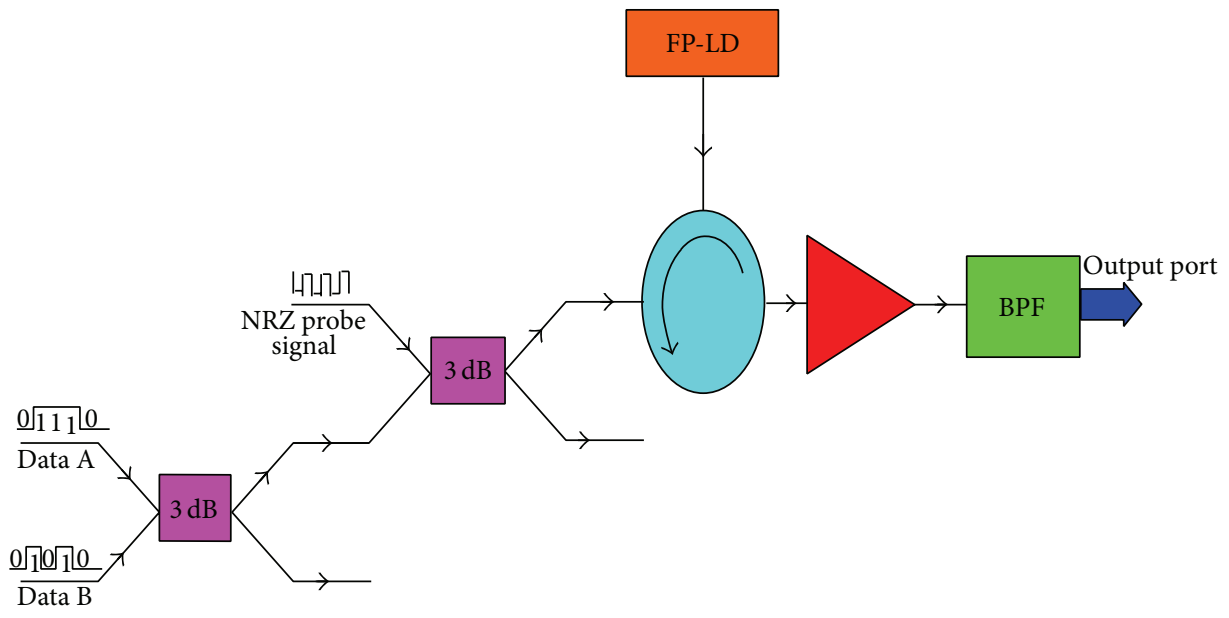

FIGURE 4: Design of gate using circulator.

is injected to the FP-LD with a detuning range higher than the previous beam, the first beam will be suppressed while the second beam will experience a gain and also FP-LD will be locked by the second beam. This is a "gain modulation" technique in which pump power should be more than probe signal [19].

2.1.4. Optical Channel-Dropping Filter. Novel design of alloptical logic AND/OR designed by using dark bright soliton conversion is shown in Figure 5. Here the dark (D) and bright (B) solitons represent the input logic "0" and " 1 ", respectively. The input of stage-1 optical channel dropping filter (OCDF) is dark soliton (logic "0") which is a control light pulse. In stage1 OCDF optical filter, the dark soliton is converted into dark and bright solitons. Next the data A is fed into stage-2 A/D filters, and then data B is fed into stage- 3 OCDF filters. Stages are divided according to the sequence of the input given. OCDF is composed with two sets of coupled waveguides which produce a phase shift of $\pi$ with respect to the input signal [20].

2.1.5. Multilayer Waveguide. Several all-optical devices using optical nonlinearity have been proposed and implemented. In the design a multilayer planer waveguide with nonlinear guided film is taken as shown in Figure 6. The waveguide is divided in three sections $\mathrm{L}_{1}, \mathrm{~L}_{2}$, and $\mathrm{L}_{3}$ which corresponds to nonlinear three branch output, nonlinear double trapped waveguide, and linear two branch input sections, respectively. 

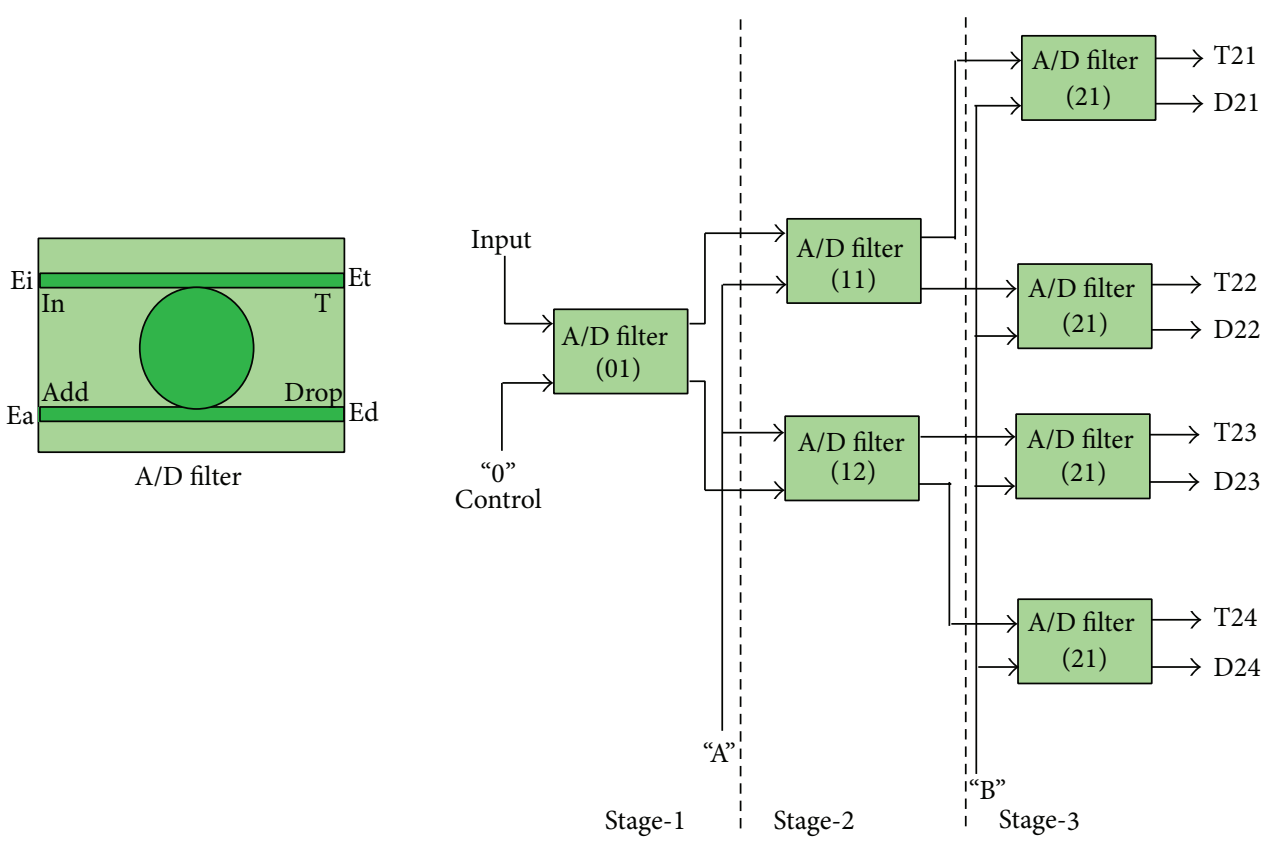

FIGURE 5: Design of gate consisting of A/D filter.

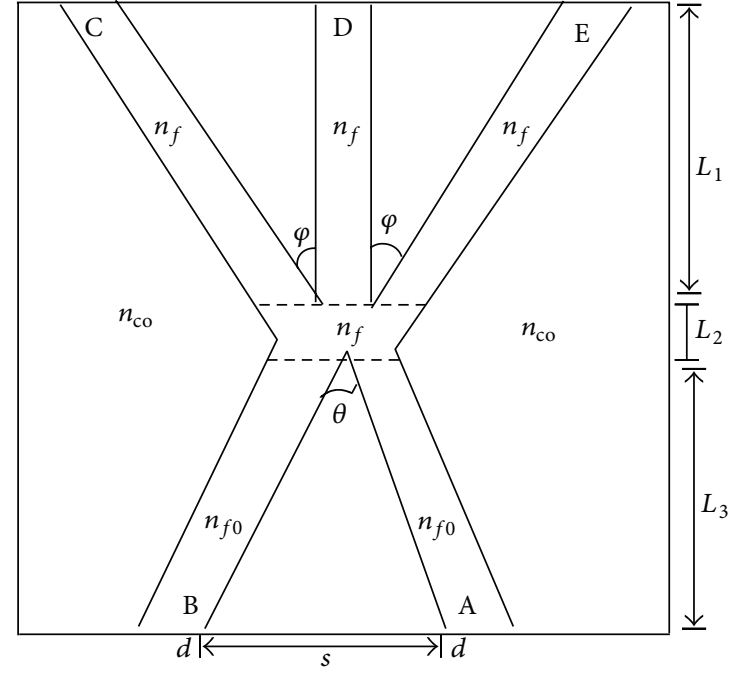

Figure 6: Gate constructed with multilayer waveguide.
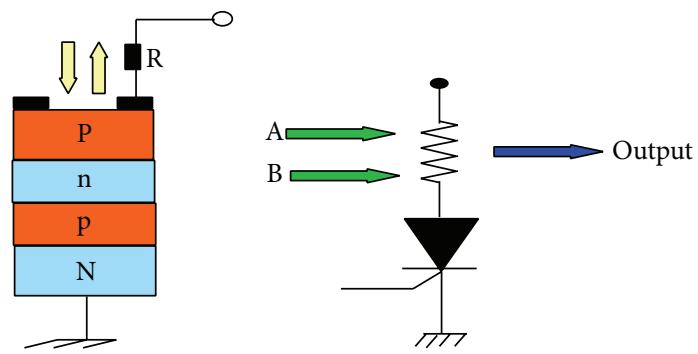

FIGURE 7: Design of gate consisting of optical thyristor.

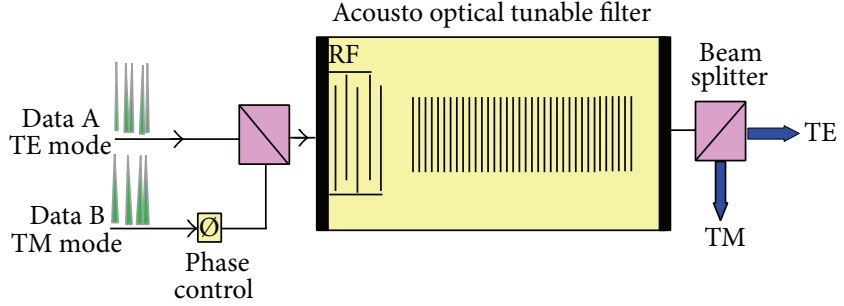

FIGURE 8: Design of gate using acoustic-optics tunable filter.

Branching angle between inputs is $\theta$ and output is $\varphi$. The refractive index of the upper three arms and lower two arms is $n_{f 0}$ and $n_{f}$, respectively. By changing the nonlinearity of the output branch and launching the input power accordingly, the optical gate can be verified [21,22].

2.1.6. Double Heterostructure Optical Thyristor. In Figure 7 authors demonstrate a monolithically integrated vertical cavity laser with depleted optical thyristor (VCL-DOT) structure which can configure into many optical logic functions using a simple operating technique by changing the condition of the driving voltage. As in Figure 7 is a bistable PnpN active region device. If the forward bias is applied to the thyristor, the s-shaped current-voltage characteristics are divided in three distinct stages, forward blocking region, negative resistance region, and forward conduction region. The forward conduction region is known as $\mathrm{ON}$-state and the forward blocking region is known as OFF-state for the optical thyristor. Boolean optical gates can be realized by connecting these thyristors in series or in parallel and changing the reference voltage [23]. 


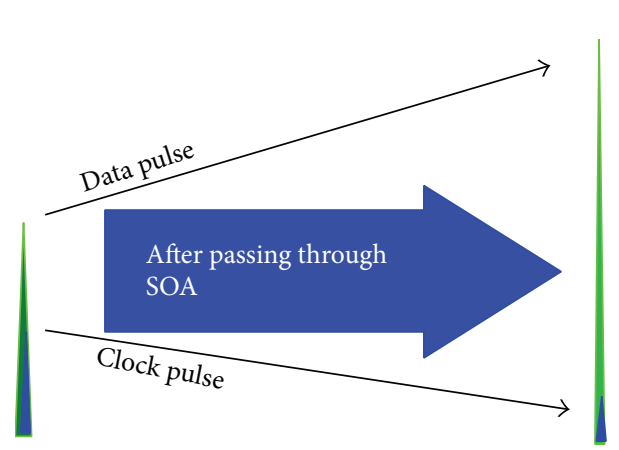

(a)

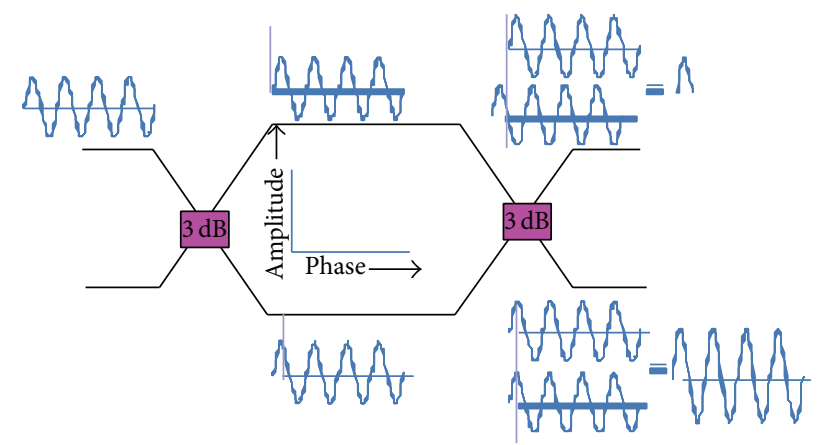

(b)

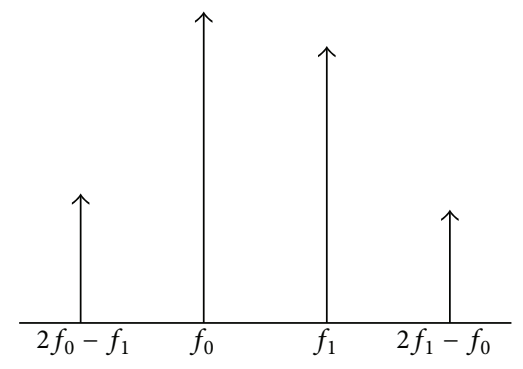

(c)

FIGURE 9: Nonlinearity in SOA: (a) XGM, (b), XPM, and (c) FWM.

2.1.7. Acousto Optical Tunable Filter (AOTF). Operation of gates is based on the switching of TE and TM polarized data $\mathrm{A}_{\mathrm{TE}}$ and $\mathrm{B}_{\mathrm{TM}}$ and vice versa as in Figure 8. The polarized data are then modulated in pulse position modulation (PPM). Then the polarized data are excited through SAW transducer. Pulses pass through acoustic-optic waveguide that determine the wavelength to be transmitted. The Boolean logic gates are verified by the temporal displacement of the output pulse [24].

2.2. All-Optical Gates with SOA. Again the gates are divided according to the interferometer techniques such as ultra-high nonlinear interferometer (UNI), sagnac interferometer (SI), Michelson interferometer (MI), Mach-Zehnder interferometer (MZI), and delay interferometer (DI) to implement the nonlinearity in SOA. In the following sections the nonlinearity of SOA may be used in several ways. Different design structures and categories of SOA-based all-optical gates have been investigated in this section.

SOA is a small size nonlinear amplifier that offers advantages to be integrated to produce a subsequent system essential in optical communication system. The SOAs exhibit low power consumption and their single mode waveguide structures make them particularly appropriate for use with single mode fiber [3]. At present, SOA is the most developed optical amplifier that makes a rapid progress towards optical signal processing. The nonlinearity effect in SOA makes it a promising module for optical logic gates. The three nonlinearity effects that is cross gain modulation (XGM), cross phase modulation (XPM), and four wave mixing (FWM) make it possible to use it as nonlinear medium for gates.
In XGM data pulses at one wavelength, modulates the carrier density and at the same time results as a gain variation indentation in inverted copy of the clock pulse injected into the SOA as shown in Figure 9(a). Due to the modulation of a carrier density there is a gain compression in the pump signal that produces a chirping of the converted signal. The SOA is operated under the high optical intensity to reduce the gain recovery time. The problem related to XGM is at longer wavelength extinction ratio penalty associated with it. This phenomenon can be easily accommodated at high bit rate.

The chirp of the converted signal is used as an advantage byincluding the SOA in an interferometer configuration that converts this XPM into an intensity modulation. This can be done by SOA, incorporated with interferometer configuration. To obtain a complete extinction in an interferometer a phase shift of $\pi$ is needed as in Figure 9(b), which can be achieved with gain compression in SOA. The phase shift is independent of wavelength, so the conversion to a longer wavelength has no problem with XPM. The disadvantage of an interferometer structure is that if the phase shift increases more than $\pi$, it impairs the extinction ratio which may be controlled by changing the bias condition of SOA. The interferometer configuration may be defined in two ways, copropagation and counterpropagation. In copropagation, filter is required because pump and probe travel in the same direction to filter the probe signal with pump. But in counterpropagation both travel in opposite directions, so the filter is not required.

In FWM two signals of different wavelengths are injected into the SOA. On passing through SOA there is an intensity beating which arises due to the difference in frequency modulated signals in SOA. If the frequency separation is 


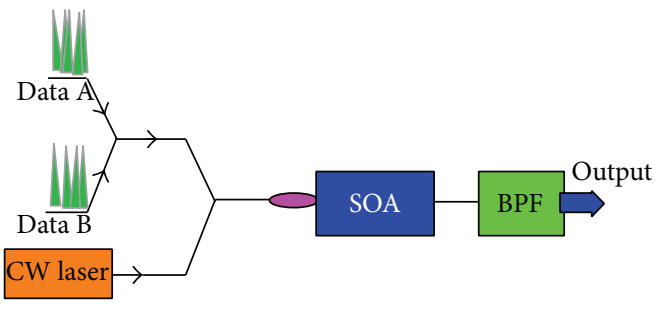

(a)

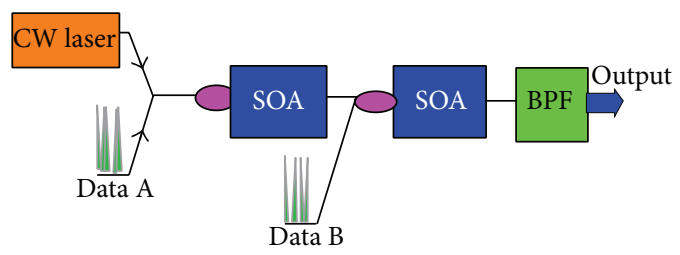

(b)

FIGURE 10: Copropagating UNI gates (a) with single SOA and (b) with dual SOA.

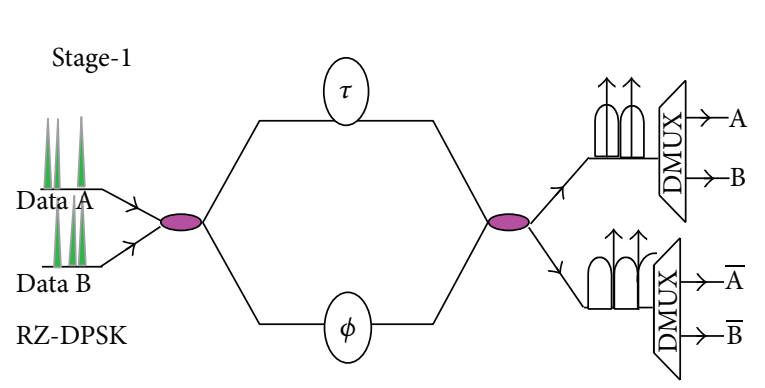

(a)

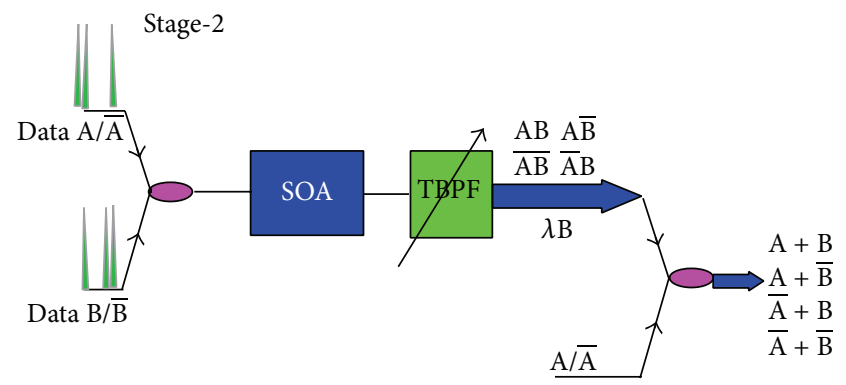

(b)

FIGURE 11: Principal of conical logic unit: (a) preprocessing unit to get data (Buffer) and complementary data (NOT); (b) unit to get minterm (AND) and maxterm (OR).

small the carrier density will be modulated. If the frequency separation is large, the modulated carrier will set up a moving grating in the active strip of SOA. The grating scatters the input signal and produces the sidebands which are located at the lower and higher frequency between the input signals. The power of the side bands is usually less as compared to the signal power as in Figure 9(c). It is a process which depends on the phase of the optical signal instead of their intensity. It is a polarization dependent phenomenon and capable of handling intensity modulation, phase modulation, and frequency shift keying signal. As it depends upon distance between the signals and converted wavelengths, therefore the conversion efficiency is adequately affected. Therefore the scheme is not used in all-optical network. The application of FWM is used in dispersion management by optical phase conjugate. The process produces a mirror image of the original signal which is oppositely chirped in a spectral domain [25].

\subsubsection{Ultranonlinear Interferometer (UNI) Configuration.} The concept of operation of the UNI gates relies on polarization rotation of the incoming signals to be switched in the presence of a switched pulse in SOA. It is divided in two copropagating UNI gates and counterpropagating UNI gates.

(1) UNI Copropagating Gates. According to the design structure UNI copropagating gates can be divided in two categories. In the first design, the data and clock directly inter into SOA and on the other hand the data is first modulated through delay interferometer (DI) and then send to SOA.

(i) UNI Copropagating Gates. Figure 10(a) shows the basic operation of UNI gates that depends on the differential phase

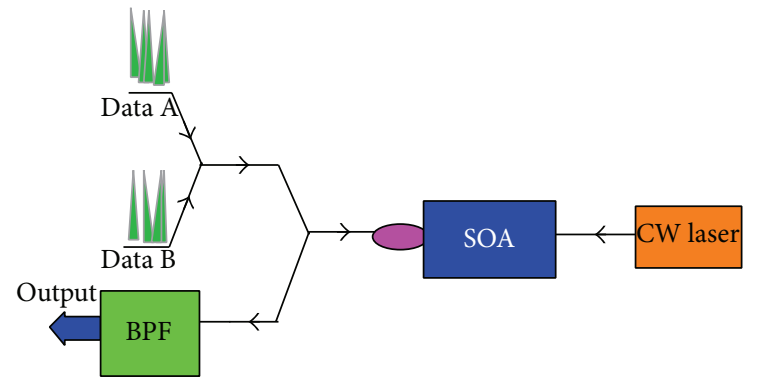

FIGURE 12: Counterpropagating UNI gate.

shift between two orthogonal polarized components of the signal [26-37]. Here the clock pulse is orthogonally polarized and delayed after passing through polarization maintained fiber (PMF). The phase and amplitude of modulated data with higher power are copropagated through SOA. If both the data are present or absent the differential phase shift between the probe signals includes destructive interference; therefore output will be zero. If only one data is present, the phase change in the probe is adjusted to introduce constructive interference at the output. Another technique of dual SOAUNI gate is shown in Figure 10(b). It may be constructed with two SOAs in UNI based elements [38].

(ii) All-Optical Conical Logic Unit. The operational principal of the design is divided into two stages as shown in Figure $11[39,40]$. In stage-1 the carrier wavelength of DPSK signals is sent simultaneously to delay interferometer (DI). $\mathrm{DI}$ is an asymmetric Mach-Zehnder interferometer (MZI) with differential delay and tunable phase shift in both the 


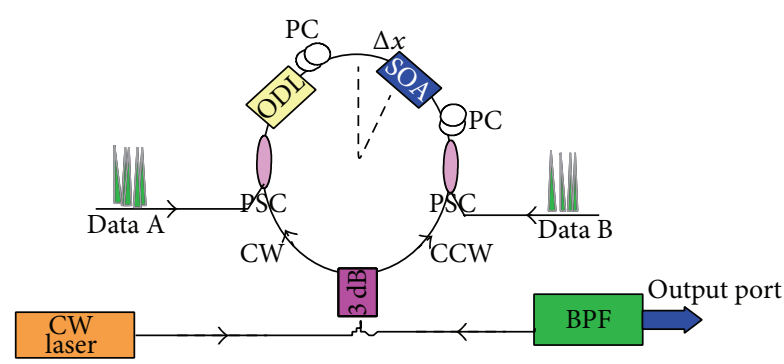

FIGURE 13: Sagnac interferometer gate.

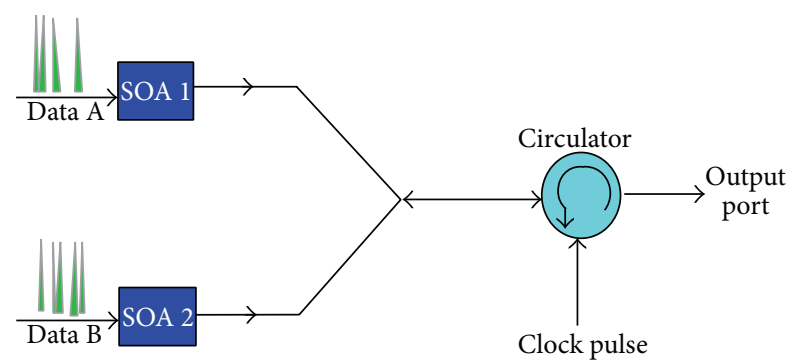

FIGURE 14: Michelson interferometer gate.

arms, respectively. When DPSK signals pass through DI, constructive and destructive signals are created, that are separated through wavelength division multiplexing (WDM) to get Buffer and NOT operation at both the output ports, respectively. The output of DI is then launched into stage2. In stage- 2 both the data are injected into the SOA, out of which one acts as a pump and other as a probe. After passing through SOA, there is a cross gain modulation (XGM) and the output that is minterms $\left(m_{2}\right)$ or AND operation is selected accordingly at the output of tunable band pass filter (TBPF). Again, the full set of minterms are combined together with original data directly to obtain maxterm $\left(M_{2}\right)$ or OR operation.

(2) UNI Counterpropagating Gates. Figure 12 shows the counterpropagation in UNI gates where the clock and data signal propagate in opposite directions. Therefore the pump signal passes through SOA causing the carrier depletion in SOA. The carrier depletion leads to gain saturation in SOA. Due to this, there is a marked intensity reduction of an incoming probe signal, which leads to no pulse existence for an output signal $[41,42]$. If two SOAs are used in parallel, the output of first can be used to construct the multifunctional logic gates.

2.2.2. Sagnac Interferometer (SI) Gates. The design as in Figure 13 consists of optical fiber loop with SOA placed asymmetrically. This gate is using the principle of TOAD (terahertz optical asymmetric demultiplexer). The offset position of SOA is controlled to obtain short switching window using nonlinearity in SOA. The sagnac interferometer gate consists of a $2 \times 2$ coupler which is used to join input port and output port. To maintain the polarization state of the fiber, polarization controller $(\mathrm{PC})$ is used with polarization maintained fiber (PMF). The clock signal propagates through

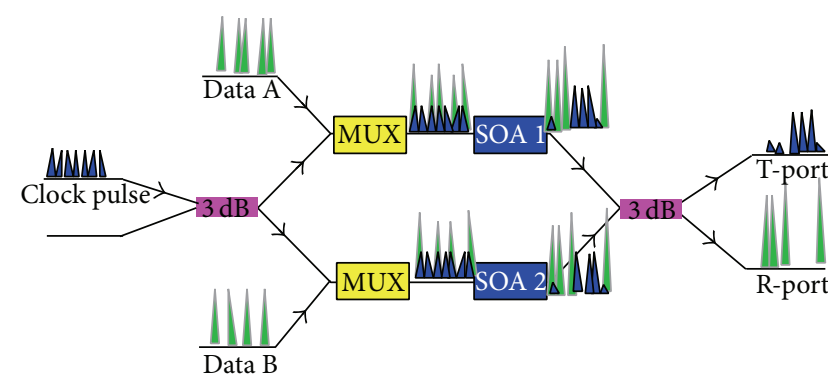

FIGURE 15: Copropagation Mach-Zehnder interferometer gate.

the coupler and splits in two equal parts with phase difference of $\pi / 2$. One will travel in clockwise (CW) direction and the other will travel in counter-clockwise direction (CCW) in the fiber loop. The time asymmetry between CW and CCW is maintained at $\Delta x$. Orthogonally polarized data enters through the polarization selective coupler (PSC) into the fiber loop. After passing through SOA there is a XPM between two counterpropagating probe signals. If any data is present due to differential phase shift, two probes interfere constructively on looping back to the coupler resulting as one. If both the data are present or absent the two probes interfere destructively and the output become zero [43-50].

2.2.3. Michelson Interferometer (MI) Gates. The MI arrangement as depicted in Figure 14 is half or folded version of MZI of the counterpropagation scheme. The SOA is placed at the upper and lower arm of MI. When data of same wavelengths $\mathrm{A}$ and $\mathrm{B}$ passes through both SOAs in the opposite directions of clock wave signal, the refractive index produces phase variation in the medium of SOA. This modulates the clock wave signal incorporating the phase modulation at the output terminal. The circulator is used to recombine the reflected clock wave from both the SOAs. When constructive interference between two interferometer paths is maintained, the circulator output produces a converted signal. And destructive interference between two interferometer paths is maintained; the circulator produces no signal. It comprises a simple structure utilizing only one coupler, smaller in size, and requires less signal power than MZI [51-53].

\subsubsection{Mach-Zehnder Interferometer (MZI) Configuration.} There are several possibilities for realizing the optical gates utilizing XPM in SOA based interferometer configuration. The gates comprise two SOAs located in the two paths or two arms, in which phase to amplitude modulation can be obtained when a relative phase difference is introduced in the interferometer. This phase difference may be produced in various ways, such as using $2 \times 2$ coupler, inserting a phase shifter in both the arms, or using different values of SOA, and so forth.

(1) MZI Copropagation. The MZI configuration may be divided into two categories that are MZI in copropagation and MZI in counterpropagation. In MZI copropagation, probe and pump propagate in the same direction. Thus, in 


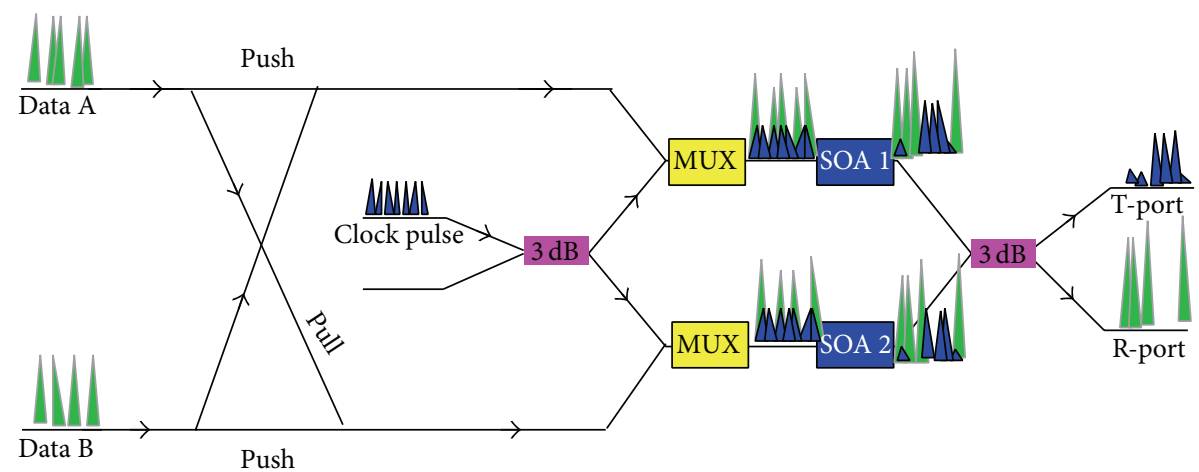

FIgURE 16: Mach-Zehnder interferometer with push-pull configuration.

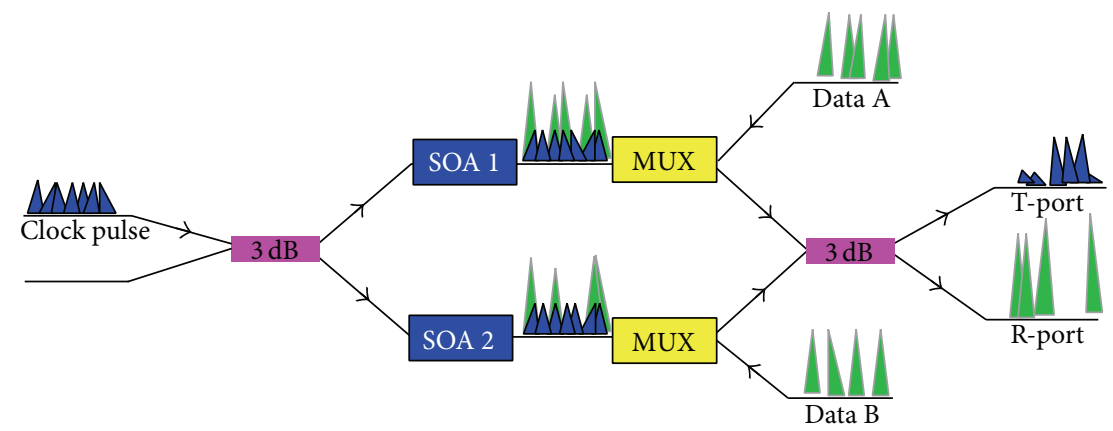

FIGURE 17: Counterpropagating MZI gate.

such configuration band pass filter is required to filter the probe pulse from pump.

(i) MZI Copropagating Gates. Copropagation MZI is a suitable device for high speed all-optical demultiplexing. It operates on the principle of phase change, caused by the ray of light propagating through the $3 \mathrm{~dB}$ coupler. Gates consist of a symmetrical MZI with two SOAs placed in the upper and lower arm of the interferometer, as shown in Figure 15. In order to perform the operation two data streams enter in the upper and lower arms of interferometer through multiplexers. A continuous clock pulse through mode lock laser (MLL) enters through upper arm of first $3 \mathrm{~dB}$ coupler. When both pulses pass through SOA, the XGM will take place due to gain saturation in SOA. Data and clock pulses of different wavelengths are injected into SOA, operated under the gain saturation condition, where the available optical gain is distributed between two wavelengths depending on their relative photon densities. Therefore, the data is transferred in the clock pulse but in inverted form. It happens in both, that is, lower and upper arms of interferometer. After passing through first $3 \mathrm{~dB}$ coupler, the phase difference of $\pi / 2$ is created between upper and lower arms of clock pulse, which is travelling through the interferometer. When saturated pulse passes through second $3 \mathrm{~dB}$ coupler the total phase shift becomes $\pi$. If both the data have same value, they will cancel because of $\pi$ phase difference; therefore, at transmission port zero will appear. If both the data have different value then it will not cancel; therefore, one will appear at transmission port [54-64].

(ii) MZI Copropagating Push-Pull Gates. The principle of operation of push-pull configuration is shown in Figure 16. Two data streams A and B of same wavelengths are entered through upper and lower arm of MZI. The data A in the upper arm is ahead of one bit period to data B which is travelling in the lower arm. Similarly, lower arm data B is one bit period ahead to upper arm data A. This creates a switching window for data streams. Both the data are copropagated with clock pulse which enters through $3 \mathrm{~dB}$ coupler. When data $\mathrm{A}$ is 1 and $\mathrm{B}$ is zero, the pulse from data A splits into two parts. One pulse is pushed to the upper SOA and other is delayed by the switching window. Thus, the upper SOA is switched before the lower SOA. Therefore, the MZI is unbalanced and clock wave is switched at the T-port. If data A is zero and data $\mathrm{B}$ is one, then lower SOA is switched and again clock wave appears at T-port. If both the data are the same, then the SOAs are equally affected by the injected pulse. Consequently, the respective push and pull pulse temporarily coincide with each other and zero phase difference is introduced between the two arms. Thus, no switching occurs at T-port [65-70].

(2) MZI Counterpropagating Gates. In counterpropagation the clock and data pulse propagate in opposite direction through MZI as in Figure 17. If any of the data is one, there is a XPM between the clock and data pulse inside SOA that creates the differential phase shift between the two clock 
TABLE 1: Comparison between non-SOA based gates.

\begin{tabular}{|c|c|c|c|c|c|c|c|}
\hline $\begin{array}{l}\text { Reported gates } \\
\text { category }\end{array}$ & $\begin{array}{l}\text { Reported } \\
\text { gates }\end{array}$ & $\begin{array}{c}\text { Contrast } \\
\text { ratio/extinction } \\
\text { ratio }\end{array}$ & $\begin{array}{l}\text { Operating } \\
\text { speed }\end{array}$ & $\begin{array}{c}\text { Modulation } \\
\text { type }\end{array}$ & $\begin{array}{l}\text { Nonlinear } \\
\text { element }\end{array}$ & $\begin{array}{c}\text { Polarization } \\
\text { sensitivity }\end{array}$ & $\begin{array}{c}\text { Integration } \\
\text { capacity }\end{array}$ \\
\hline HNLF/DSF & $\begin{array}{c}\text { XOR/OR/NOT/ } \\
\text { AND/NXOR/ } \\
\text { NOR/XNOR }\end{array}$ & $\begin{array}{c}20 / 24.25 / 30 / 1 \\
6.5 \mathrm{~dB}\end{array}$ & $40 \mathrm{G} / 10 \mathrm{~Gb} / \mathrm{s}$ & $\begin{array}{c}\text { FWM/XPM } \\
\text { /SPM/NPR }\end{array}$ & $\begin{array}{c}\text { DSF/ } \\
\text { HNLF-DSF/ } \\
\text { DSF }\end{array}$ & More & $\mathrm{Bad}$ \\
\hline $\begin{array}{l}\text { Waveguide } \\
\text { configuration }\end{array}$ & $\begin{array}{c}\text { XOR/NOR/ } \\
\text { NOT/NAND/ } \\
\text { AND }\end{array}$ & $30 / 20 \mathrm{~dB}$ & $40 \mathrm{G} / 100 \mathrm{Mb} / \mathrm{s}$ & FWM/TFA & $\begin{array}{l}\text { FP Chip/ } \\
\text { waveguide }\end{array}$ & More & Moderate \\
\hline Circulator & NOR/NOT & - & $10 \mathrm{~Gb} / \mathrm{s}$ & $\begin{array}{c}\text { Gain } \\
\text { modulation }\end{array}$ & FP-LD & More & Moderate \\
\hline $\begin{array}{l}\text { Optical } \\
\text { channel-Dropping } \\
\text { filter }\end{array}$ & AND/OR & - & - & $\begin{array}{c}\text { Dark-bright } \\
\text { solitons }\end{array}$ & OCDF & No & Moderate \\
\hline $\begin{array}{l}\text { Multibranch } \\
\text { waveguide }\end{array}$ & AND/OR & - & - & $\begin{array}{c}\text { Localized } \\
\text { optical } \\
\text { non-linearity }\end{array}$ & $\begin{array}{c}\text { Multibranch } \\
\text { non-linear } \\
\text { media }\end{array}$ & More & Compact \\
\hline $\begin{array}{l}\text { Double } \\
\text { heterostructure } \\
\text { optical thyristor }\end{array}$ & $\mathrm{AND} / \mathrm{OR}$ & $50 \mathrm{~dB}$ & - & - & VCL-DOT & No & Compact \\
\hline AOTF & AND/OR & - & $300 \mathrm{~Gb} / \mathrm{s}$ & SPM & AOTF & More & Compact \\
\hline
\end{tabular}

TABLE 2: Comparison between SOA based gates.

\begin{tabular}{|c|c|c|c|c|c|c|c|}
\hline $\begin{array}{l}\text { Reported } \\
\text { gates } \\
\text { category }\end{array}$ & $\begin{array}{l}\text { Reported } \\
\text { gates }\end{array}$ & $\begin{array}{l}\text { Extinction } \\
\text { ratio }(\mathrm{dB})\end{array}$ & $\begin{array}{l}\text { Operating } \\
\text { speed } \\
(\mathrm{Gb} / \mathrm{s}) \\
\end{array}$ & $\begin{array}{l}\text { Modulation } \\
\text { type }\end{array}$ & $\begin{array}{l}\text { Nonlinear } \\
\text { element }\end{array}$ & $\begin{array}{c}\text { Polarization } \\
\text { sensitivity }\end{array}$ & $\begin{array}{c}\text { Integration } \\
\text { capacity }\end{array}$ \\
\hline UNI copropagating & $\begin{array}{c}\text { XOR/AND/NOR/ } \\
\text { OR/NOT/ } \\
\text { XNOR/NAND } \\
\end{array}$ & $8 / 10 / 11$ & $5 / 10 / 20 / 40$ & XGM, FWM & SOA & More & Compact \\
\hline $\begin{array}{l}\text { UNI } \\
\text { counterpropagating }\end{array}$ & $\begin{array}{c}\text { AND/NOR/OR/X } \\
\text { NOR }\end{array}$ & 6.5 & 10 & XGM, FWM & SOA & More & Compact \\
\hline $\begin{array}{l}\text { Sagnac } \\
\text { interferometer }\end{array}$ & $\begin{array}{c}\text { XOR/NOT/AND/ } \\
\text { OR }\end{array}$ & $21.12 / 14.7 / 22$ & $10 / 40 / 100$ & XGM & SOA & No & Moderate \\
\hline $\begin{array}{l}\text { Michelson } \\
\text { Interferometer }\end{array}$ & XOR & 11 & 10 & XGM & SOA & No & Compact \\
\hline $\begin{array}{l}\text { MZI } \\
\text { configuration }\end{array}$ & $\begin{array}{c}\text { XOR/XNOR/AND } \\
\text { /NAND/OR/ } \\
\text { NOR } \\
\end{array}$ & $15 / 18 / 30$ & $10 / 20 / 40 / 80$ & XGM, XPM & SOA & No & Compact \\
\hline $\begin{array}{l}\text { MZI } \\
\text { Push-pull } \\
\text { Configration }\end{array}$ & $\mathrm{XOR} / \mathrm{OR}$ & $7.8 / 11 / 12$ & $10 / 20 / 40$ & XGM, XPM & SOA & No & Moderate \\
\hline $\begin{array}{l}\text { MZI counter } \\
\text { Propagating }\end{array}$ & $\begin{array}{c}\text { XOR/NOR/ } \\
\text { XNOR }\end{array}$ & $8 / 9.22 / 30$ & $10 / 40$ & XGM, XPM & SOA & No & Compact \\
\hline $\begin{array}{l}\text { DI } \\
\text { configuration }\end{array}$ & $\begin{array}{l}\text { OR/NOR/ } \\
\text { XOR }\end{array}$ & 13.9 & 40 & XGM, XPM & SOA & No & Moderate \\
\hline
\end{tabular}

components which unbalance the MZI and the clock pulse exits at the T-port. However, if both the data are the same, the total phase shift will become $\pi$ and clock pulse is cancelled at T-port. In this configuration no filter is required as data and clock pulse counterpropagate through the arm of MZI. Thus, at T-port there is no data pulse to filter [71-74].

2.2.5. Delayed Interferometer (DI) Gates. The delay interferometer (DI) in Figure 18, the clock, and two data pulses of same wavelengths are injected into the SOA simultaneously $[75,76]$. The data pulses will include a XGM, which leads to invert the clock signal in SOA. Then, the clock enters into DI and splits into two signals propagating through the two arms of DI with equal amplitude which interferes at the output. One arm of DI is delayed by $\Delta t$ relative to the other arm. Therefore, at the output there is a relative phase difference between the two signals that interferes and produces an output. The XPM phenomenon produces a time-varying 


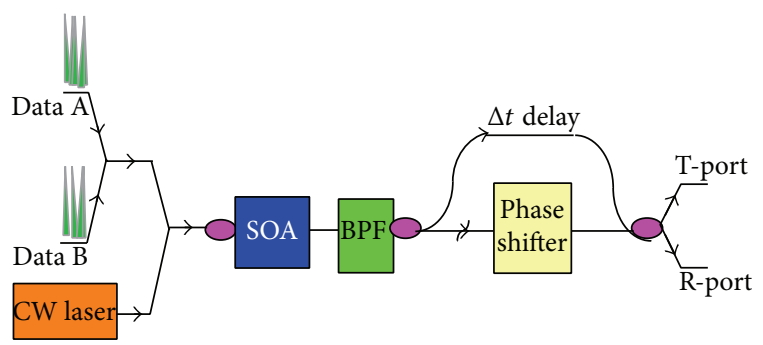

FIGURE 18: Delayed interferometer gate.

phase change on the clock pulse. The delay $\Delta t$ determines the speed and the signal-to-noise ratio needed for good signal quality. A low $\Delta t$ would restrict the speed of the data processing and also degrade the signal to noise ratio.

\section{Conclusion}

As discussed in the paper, all-optical gates of different designs have their utility but still the competitors are digital gates, which are compact and easy in coupling. If one goes for speed, optical gates are leading but in the case of extinction ratio (ER) the digital gates are still superior. The different designs use different nonlinear elements to create modulations. In Tables 1 and 2, different design structures are compared according to the polarization sensitivity and integration capacity. The nonlinear elements used in optical gates are length of the fiber, crystal waveguide, circulators, filters, thyristor, acoustic tunable filter and SOAs as discussed above in different schemes. Some optical gates such as HNLF/DSF, sagnac interferometer and delay interferometer configurations are bigger that make it inconvenient as compared to digital gates. Some gates using circulators, optical channeldropping filters, thyristor, and acoustic tunable filter have been reported less number of logical gates but still they are compact in size. The MZI copropagation waveguide structure design reported all types of logical gates with high operating speed and good extinction ratio that makes them superior than others.

\section{Conflict of Interests}

The authors declare that there is no conflict of interests regarding the publication of this paper.

\section{References}

[1] I. P. Kaminow and T. L. Koch, Optical Fiber Telecommunications IIIA, Academic Press, 1997.

[2] G. P. Agrawal, Nonlinear Fiber Optics, Academic Press, 4th edition, 2007.

[3] J. M. Senior, Optical Fiber Communication, Pearson, 3rd edition, 2010.

[4] A. Bogoni, L. Potì, R. Proietti, G. Meloni, F. Ponzini, and P. Ghelfi, "Regenerative and reconfigurable all-optical logic gates for ultra-fast applications," Electronics Letters, vol. 41, no. 7, pp. 435-436, 2005.
[5] L. Huo, C. Lin, C. K. Chan, and L. K. Chen, "A reconfigurable all-optical AND/OR logic gate using multilevel modulation and self phase modulation," in Proceedings of the Optical Fiber Communication and the National Fiber Optic Engineers (OFC/NFOEC '07), pp. 1-3, Anaheim, Calif, USA, 2007.

[6] B.-E. Olsson and P. A. Andrekson, "Polarization-independent all-optical AND-gate using randomly birefringent fiber in a nonlinear optical loop mirror," in Proceedings of the IEEE Optical Fiber Communication Conference (OFC '98), pp. 375376, San Jose, Calif, USA, February 1998.

[7] D. M. F. Lai, C. H. Kwok, T. I. Yuk, and K. K. Y. Wong, "Picosecond all-optical logic gates (XOR, OR, NOT, and AND) in a fiber optical parametric amplifier," in Proceedings of the IEEE Optical Fiber Communication/National Fiber Optic Engineers Conference (OFC/NFOEC '08), pp. 1-3, San Diego, Calif, USA, February 2008.

[8] E. A. M. Fagotto and M. L. F. Abbade, "All-optical demultiplexing of 4-ASK optical signals with four-wave mixing optical gates," Optics Communications, vol. 283, no. 6, pp. 1102-1109, 2010.

[9] W. Wang, J. Yu, B. Han et al., "Experimental research on $10 \mathrm{~Gb} / \mathrm{s}$ all-optical logic gates with return-to-zero data in high nonlinear fiber," in Optoelectronic Materials and Devices VI, vol. 8308 of Proceedings of SPIE, pp. 1-6, Tianjin, China, November 2011.

[10] J. Qiu, K. Sun, M. Rochette, and L. R. Chen, "Reconfigurable alloptical multilogic gate (xor,AND, and or) based on cross-phase modulation in a highly nonlinear fiber," Photonics Technology Letters, vol. 22, no. 16, pp. 1199-1201, 2010.

[11] J. Wang, Q. Sun, J. Sun, and X. Zhang, "Experimental demonstration on $40 \mathrm{Gbit} / \mathrm{s}$ all-optical multicasting logic XOR gate for NRZ-DPSK signals using four-wave mixing in highly nonlinear fiber," Optics Communications, vol. 282, pp. 2615-2619, 2009.

[12] L. Li, J. Wu, J. Qiu et al., "Reconfigurable all-optical logic gate using four-wave mixing (FWM) in HNLF for NRZ-PolSK signal," Optics Communications, vol. 283, pp. 3608-3612, 2010.

[13] B. Liu, H. Cai, X. M. Zhang, J. Tamil, Q. X. Zhang, and A. Q. Liut, "MEMS optical logic nor gate using integrated tunable laser," in Proceedings of the IEEE 22nd International Conference on Micro Electro Mechanical Systems (MEMS '09), pp. 971-974, Sorrento, Italy, 2009.

[14] K. Mukherjee and D. Kumbhakar, "Simulation of two photon absorption in silicon wire waveguide for implementation of all optical logic gates," Optik, vol. 123, no. 6, pp. 489-493, 2012.

[15] T. D. Vo, J. Schröder, R. Pant et al., "Photonic chip based alloptical XOR gate for phase-encoded signals," in Proceedings of the Optical Fiber Communication Conference and Exposition and the National Fiber Optic Engineers Conference (OFC/NFOEC '11), Los Angeles, Calif, USA, March 2011.

[16] J. Wang, J. Sun, and Q. Sun, "Proposal for all-optical switchable OR/XOR logic gates using sum-frequency generation," Photonics Technology Letters, vol. 19, pp. 541-543, 2007.

[17] A. Bogoni, X. Wu, Z. Bakhtiari, S. Nuccio, and A. E. Willner, "640 Gbits/s photonic logic gates," Optics Letters, vol. 35, pp. 3955-3957, 2010.

[18] J. Wang, J. Sun, X. Zhang, D. Huang, and M. M. Fejer, "Ultrafast all-optical three-input Boolean XOR operation for differential phase-shift keying signals using periodically poled lithium niobate," Optics Letters, vol. 33, pp. 1419-1421, 2008.

[19] M. R. Uddin, J. S. Cho, and Y. H. Won, "All-optical NOR and NOT gates at $10 \mathrm{~Gb} / \mathrm{s}$ based on gain modulation in fabry-perot laser diode," in Proceedings of the 7th International Conference on Optical Internet (COIN '08), pp. 1-2, Tokyo, Japan, 2008. 
[20] P. Phongsanam, C. Teeka, R. Jomtarak, S. Mitatha, and P. P. Yupapin, "All-optical logic AND and OR gates generated by dark-bright soliton conversion," Optik, vol. 124, pp. 406-410, 2013.

[21] Y. D. Wu, "All-optical logic gates by using multibranch waveguide structure with localized optical nonlinearity," IEEE Journal of Quantum Electronics, vol. 11, pp. 307-312, 2005.

[22] Y. M. Geshiro, T. Kitamura, K. Nishida, and S. Sawa, "All-optical logic gates containing a two-mode nonlinear waveguide," IEEE Journal of Quantum Electronics, vol. 38, pp. 37-46, 2002.

[23] W. Choi, D. G. Kim, Y. T. Moon et al., "Optical logic gates based on integrated vertical cavity laser with depleted optical thyristor structure," in Proceedings of the IEEE Nanotechnology Materials and Devices Conference (NMDC '06), pp. 330-331, Gyeongju, South Korea, 2006.

[24] C. S. Sobrinho, C. S. N. Rios, S. P. Marciano et al., "A performance study of a logical gate using PPM optical pulse modulation for TDM systems," Optics Communications, vol. 275, pp. 476-485, 2007.

[25] I. Kaminow and T. Li, Optical Fiber Communication IVA, Academic Press, 2002.

[26] J. Dong, X. Zhang, Y. Wang, J. Xu, and D. Huang, "40 Gbit=s reconfigurable photonic logic gates based on various nonlinearities in single SOA," Electronics Letters, vol. 43, pp. 884-886, 2007.

[27] S. Kumar and A. E. Willner, "Simultaneous four-wave mixing and cross-gain modulation for implementing an all-optical XNOR logic gate using a single SOA," Optics Express, vol. 14, pp. 5092-5097, 2006.

[28] J. Dong, X. Zhang, J. Xu, and D. Huang, “40 Gb/s all-optical logic NOR and OR gates using a semiconductor optical amplifier: experimental demonstration and theoretical analysis," Optics Communications, vol. 281, pp. 1710-1715, 2008.

[29] C. Bintjas, M. Kalyvas, G. Theophilopoulos et al., " $20 \mathrm{~Gb} / \mathrm{s}$ alloptical XOR with UNI gate," Photonics Technology Letters, vol. 12, pp. 834-836, 2000.

[30] M. Sahafi, A. Rostami, and A. Sahafi, "All-optical high speed logic gates using SOA," Optics Communications, vol. 285, pp. 2289-2292, 2012.

[31] T. Siarkos, K. E. Zoiros, and D. Nastou, "On the feasibility of full pattern-operated all-optical XOR gate with single semiconductor optical amplifier-based ultrafast nonlinear interferometer," Optics Communications, vol. 282, pp. 2729-2740, 2009.

[32] K. E. Zoiros and T. Siarkos, "Design rules for full patternoperated all-optical XOR gate with single semiconductor optical amplifier-based ultrafast nonlinear interferometer," in Proceedings of the 7th International Symposium on Communication Systems Networks and Digital Signal Processing (CSNDSP '10), pp. 631-635, Newcastle upon Tyne, UK, 2010.

[33] B. Wu, S. Fu, J. Wua et al., "Simultaneous implementation of alloptical OR and AND logic gates for NRZ/RZ/CSRZ ON-OFFkeying signals," Optics Communications, vol. 283, pp. 349-354, 2010.

[34] A. Villafranca, I. Garcés, M. Cabezón, J. J. Martínez, D. Izquierdo, and J. Pozo, "Multiple-bit all-optical logic based on cross-gain modulation in a semiconductor optical amplifier," in Proceedings of the IEEE 12th International Conference on Transparent Optical Networks (ICTON '10), pp. 1-4, Munich, Germany, 2010.

[35] J. Xu, X. Zhang, J. Dong, D. Liu, and D. Huang, "Ultrafast alloptical AND gate based on cascaded SOAs with assistance of optical filters," Electronics Letters, vol. 43, pp. 585-586, 2007.
[36] G. Berrettini, A. Simi, A. Malacarne, A. Bogoni, and L. Potí, "Ultrafast integrable and reconfigurable XNOR, AND, NOR, and NOT photonic logic gate," Photonics Technology Letters, vol. 18, pp. 917-919, 2006.

[37] A. Sharaiha, J. Topomondzo, and P. Morel, "All-optical logic AND-NOR gate with three inputs based on cross-gain modulation in a semiconductor optical amplifier," Optics Communications, vol. 265, pp. 322-325, 2006.

[38] R. P. Webb, X. Yang, R. J. Manning, and R. Giller, "All-optical $40 \mathrm{Gbit} / \mathrm{s}$ XOR gate with dual ultrafast nonlinear interferometer," Electronics Letters, vol. 41, pp. 1396-1397, 2005.

[39] L. Lei, J. Dong, Y. Yu, S. Tan, and X. Zhang, "All-optical canonical logic units-based programmable logic array (CLUs-PLA) using semiconductor optical amplifiers," Journal of Lightwave Technology, vol. 30, pp. 3532-3539, 2012.

[40] X. Chen, Y. Yu, and X. Zhang, "All-optical logic minterms for three-input demodulated differential phase-shift keying signals at $40 \mathrm{~Gb} / \mathrm{s}$," Photonics Technology Letters, vol. 23, no. 2, pp. 118120, 2011.

[41] C. W. Son, S. H. Kim, Y. T. Byun et al., "Realization of all-optical multi-functional logic gates using semiconductor optical amplifiers," Electronics Letters, vol. 42, no. 18, pp. 1057$1058,2006$.

[42] S. H. Kim, J. H. Kim, C. W. Son et al., "Design and simulation of all-optical and gate using XGM in semiconductor optical amplifiers without input additional beam," in Proceedings of the International Conference on Numerical Simulation of Semiconductor Optoelectronic Devices (NUSOD '06), pp. 91-92, Nanyang, China, September 2006.

[43] T. Houbavlis, "10 Gbit/s all-optical Boolean XOR with SOA fibre Sagnac gate," Electronics Letters, vol. 35, no. 19, pp. 1650-1652, 1999.

[44] I. Glesk, R. J. Runser, and P. R. Prucnal, "New generation of devices for all-optical communications," Acta Physica Slovaca, vol. 51, no. 2, pp. 151-162, 2001.

[45] J. P. Sokoloff, P. R. Prucnal, I. Glesk, and M. Kane, "A terahertz optical asymmetric demultiplexer (TOAD)," Photonics Technology Letters, vol. 5, no. 7, pp. 787-790, 1993.

[46] K. E. Zoiros, G. Papadopoulos, T. Houbavlis, and G. T. Kanellos, "Theoretical analysis and performance investigation of ultrafast all-optical Boolean XOR gate with semiconductor optical amplifier-assisted Sagnac interferometer," Optics Communications, vol. 258, no. 2, pp. 114-134, 2006.

[47] T. Chattopadhyay and J. N. Roy, "Semiconductor optical amplifier (SOA)-assisted Sagnac switch for designing of all-optical tri-state logic gates," Optik, vol. 122, no. 12, pp. 1073-1078, 2011.

[48] K. Zoiros, T. Houbavlis, K. Machos et al., "10 GHz Boolean XOR with semiconductor optical amplifier fiber Sagnac gate," in Proceedings of the Lasers and Electro-Optics Conference (CLEO '99), pp. 379-380, Baltimore, Md, USA, 1999.

[49] P. Toliver, R. J. Runser, I. Glesk, and P. R. Prucnal, "Comparison of three nonlinear interferometric optical switch geometries," Optics Communications, vol. 175, no. 4, pp. 365-373, 2000.

[50] C. Feng, J. Wu, K. Xu, and J. Lin, "Simple ultrafast all-optical AND logic gate," Optical Engineering, vol. 46, no. 12, pp. 1-5, 2007.

[51] B. Mikkelsen, T. Durhuus, C. Joergensen et al., "10 Gbit/s wavelength converter realised by monolithic integration of semiconductor optical amplifiers and Michelson interferometer," in Proceedings of the 20th European Conference on Optical Communication (ECOC '94), pp. 67-70, September 1994. 
[52] J. H. Kim, Y. M. Jhon, Y. T. Byun, S. Lee, D. H. Woo, and S. H. Kim, "All-optical XOR gate using semiconductor optical amplifiers without additional input beam," Photonics Technology Letters, vol. 14, no. 10, pp. 1436-1438, 2002.

[53] K. E. Stubkjaer, "Semiconductor optical amplifier-based alloptical gates for high-speed optical processing," IEEE Journal on Selected Topics in Quantum Electronics, vol. 6, no. 6, pp. 1428$1435,2000$.

[54] P. Singh, H. K. Dixit, D. K. Tripathi, and R. Mehra, "Design and analysis of all-optical inverter using SOA-based Mach-Zehnder interferometer," Optik, vol. 124, pp. 1926-1929, 2013.

[55] P. Singh, D. K. Tripathi, S. Jaiswal, and H. K. Dixit, "Design of all-optical buffer and OR gate using SOA-MZI," Optical and Quantum Electronics, 2013.

[56] I. Kang, M. Rasras, L. Buhl et al., "All-optical XOR and XNOR operations at $86.4 \mathrm{~Gb} / \mathrm{s}$ using a pair of semiconductor optical amplifier mach-zehnder interferometers," Optics Express, vol. 17, no. 21, pp. 19062-19066, 2009.

[57] X. Ye, P. Ye, and M. Zhang, "All-optical NAND gate using integrated SOA-based Mach-Zehnder interferometer," Optical Fiber Technology, vol. 12, no. 4, pp. 312-316, 2006.

[58] G. Wang, X. Yang, and W. Hu, "All-optical logic gates for $40 \mathrm{~Gb} / \mathrm{s}$ NRZ signals using complementary data in SOA-MZIs," Optics Communications, vol. 290, pp. 28-32, 2013.

[59] J.-Y. Kim, J.-M. Kang, T.-Y. Kim, and S.-K. Han, "All-optical multiple logic gates with XOR, NOR, OR, and NAND functions using parallel SOA-MZI structures: theory and experiment," Journal of Lightwave Technology, vol. 24, no. 9, pp. 3392-3399, 2006.

[60] M. L. Nielsen, J. Mørk, T. Fjelde, and B. Dagens, "Numerical analysis of an all-optical logic XOR gate based on an active MZ interferometer," in Proceedings of the IEEE Conference on Lasers and Electro-Optics (CLEO '02), pp. 608-609, Long Beach, Calif, USA, May 2002.

[61] C. Taraphdar, T. Chattopadhyay, and J. N. Roy, "Mach-Zehnder interferometer-based all-optical reversible logic gate," Optics \& Laser Technology, vol. 42, no. 2, pp. 249-259, 2010.

[62] H. Dong, H. Sun, Q. Wang, N. K. Dutta, and J. Jaques, " 80 Gb/s All-optical logic AND operation using Mach-Zehnder interferometer with differential scheme," Optics Communications, vol. 265, no. 1, pp. 79-83, 2006.

[63] S. Ma, Z. Chen, and N. K. Dutta, "All-optical logic gates based on two-photon absorption in semiconductor optical amplifiers," Optics Communications, vol. 282, no. 23, pp. 4508-4512, 2009.

[64] C. Reis, A. Maziotis, C. Kouloumentas et al., "All-optical synchronous S-R flip-flop based on active interferometric devices," Electronics Letters, vol. 46, no. 10, pp. 709-710, 2010.

[65] R. P. Webb, R. J. Manning, G. D. Maxwell, and A. J. Poustie, " $40 \mathrm{Gbit} / \mathrm{s}$ all-optical XOR gate based on hybrid-integrated Mach-Zehnder interferometer," Electronics Letters, vol. 39, no. 1, pp. 79-80, 2003.

[66] J. M. Dailey, S. K. Ibrahim, R. J. Manning et al., " 42.6 Gbit/s fully integrated all-optical XOR gate," Electronics Letters, vol. 45, no. 20, pp. 1047-1049, 2009.

[67] M. Suzuki and H. Uenohara, "Investigation of all-optical error detection circuit using SOA-MZI-based XOR gates at $10 \mathrm{Gbit} / \mathrm{s}$," Electronics Letters, vol. 45, pp. 224-225, 2009.

[68] Q. Wang, G. Zhu, H. Chen et al., "Study of all-optical XOR using Mach-Zehnder interferometer and differential scheme," IEEE Journal of Quantum Electronics, vol. 40, pp. 703-710, 2004.
[69] Y. Aikawa, S. Shimizu, and H. Uenohara, "Demonstration of all-optical divider circuit using SOA-MZI-type XOR gate and feedback loop for forward error detection," Journal of Lightwave Technology, vol. 29, pp. 2259-2266, 2011.

[70] J. M. Dailey, R. P. Webb, and R. J. Manning, "All-optical technique for modulation format conversion from on-off-keying to alternate mark-inversion," Optics Express, vol. 18, pp. 2187321882, 2010.

[71] R. Vilar, J. M. Martinez, F. Ramos, and J. Marti, "All-optical DGD monitor for packet-switched networks based on an integrated active Mach-Zehnder interferometer operating as logic XOR gate," Optics Communications, vol. 281, pp. 53305334, 2008.

[72] S. Lee, J. Park, K. Lee, D. Eom, S. Lee, and J. H. Kim, "All-optical exclusive NOR logic gate using Mach-Zehnder interferometer," Japanese Journal of Applied Physics, vol. 41, pp. 1155-1157, 2002.

[73] M. Zhang, Y. Zhao, L. Wang, J. Wang, and P. Ye, "Design and analysis of all-optical XOR gate using SOA-based MachZehnder interferometer," Optics Communications, vol. 223, pp. 301-308, 2003.

[74] T. Houbavlis, K. E. Zoiros, G. Kanellos, and C. Tsekrekos, "Performance analysis of ultrafast all-optical Boolean XOR gate using semiconductor optical amplifier-based Mach-Zehnder interferometer," Optics Communications, vol. 232, pp. 179-199, 2004.

[75] X. Zhao, C. Lou, and Y. Feng, "Optical signal processing based on semiconductor optical amplifier and tunable delay interferometer," Optoelectron, vol. 4, pp. 308-314, 2011.

[76] D. Kong, Y. Li, H. Wang et al., "All-optical XOR gates for QPSK signals based on four-wave mixing in a semiconductor optical amplifier," Photonics Technology Letters, vol. 24, pp. 988-990, 2012. 

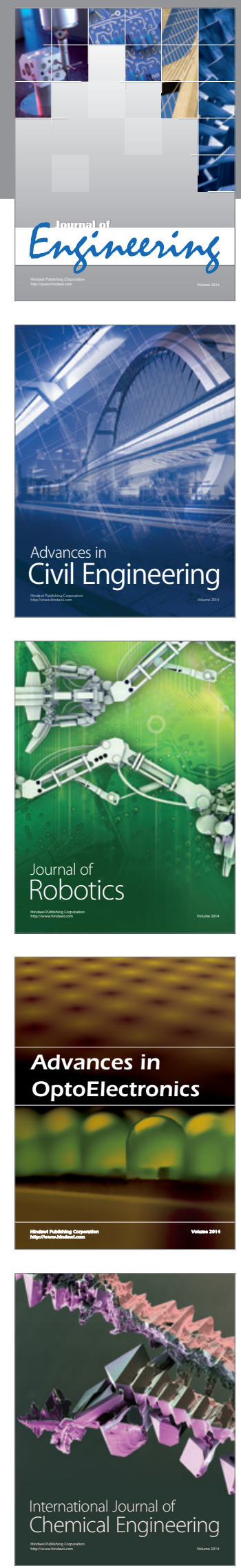

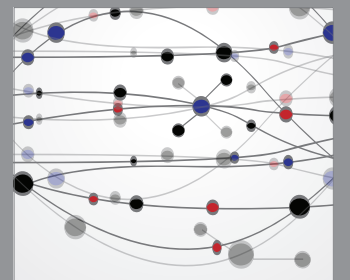

The Scientific World Journal
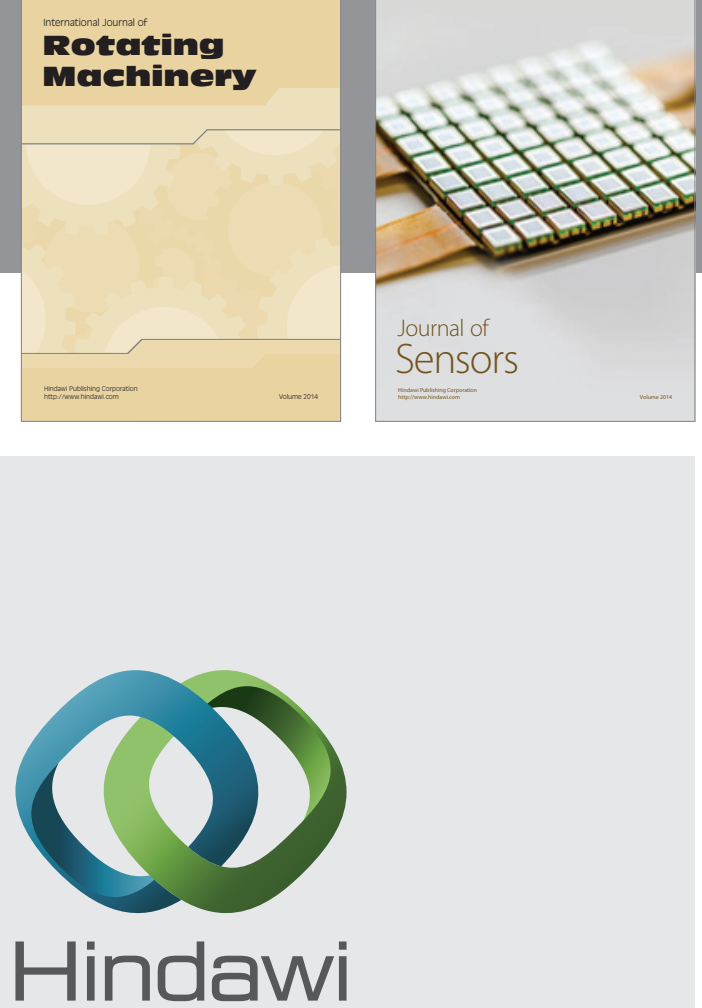

Submit your manuscripts at http://www.hindawi.com
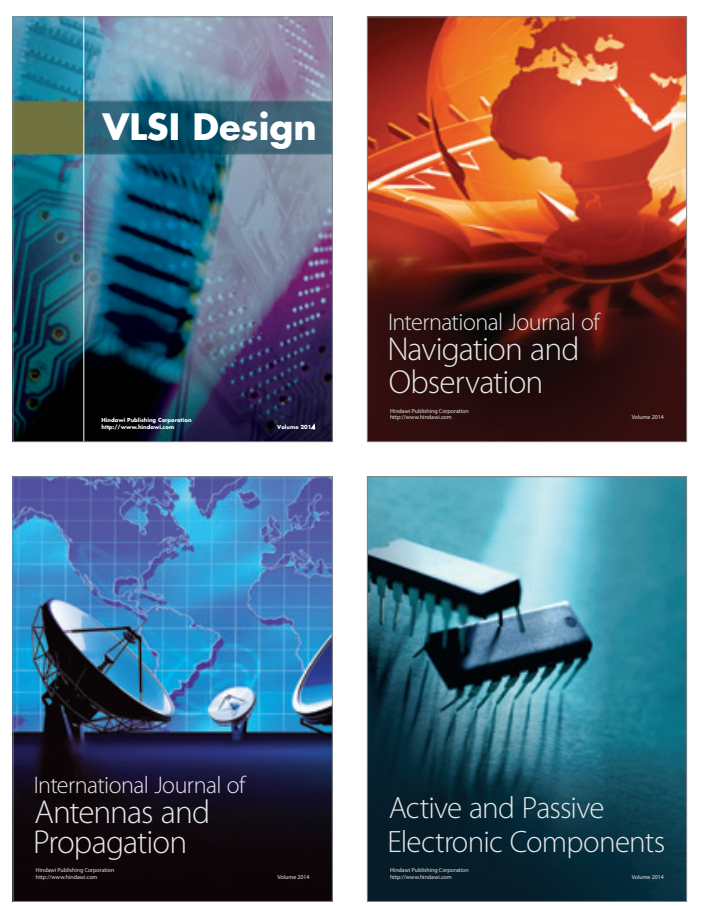
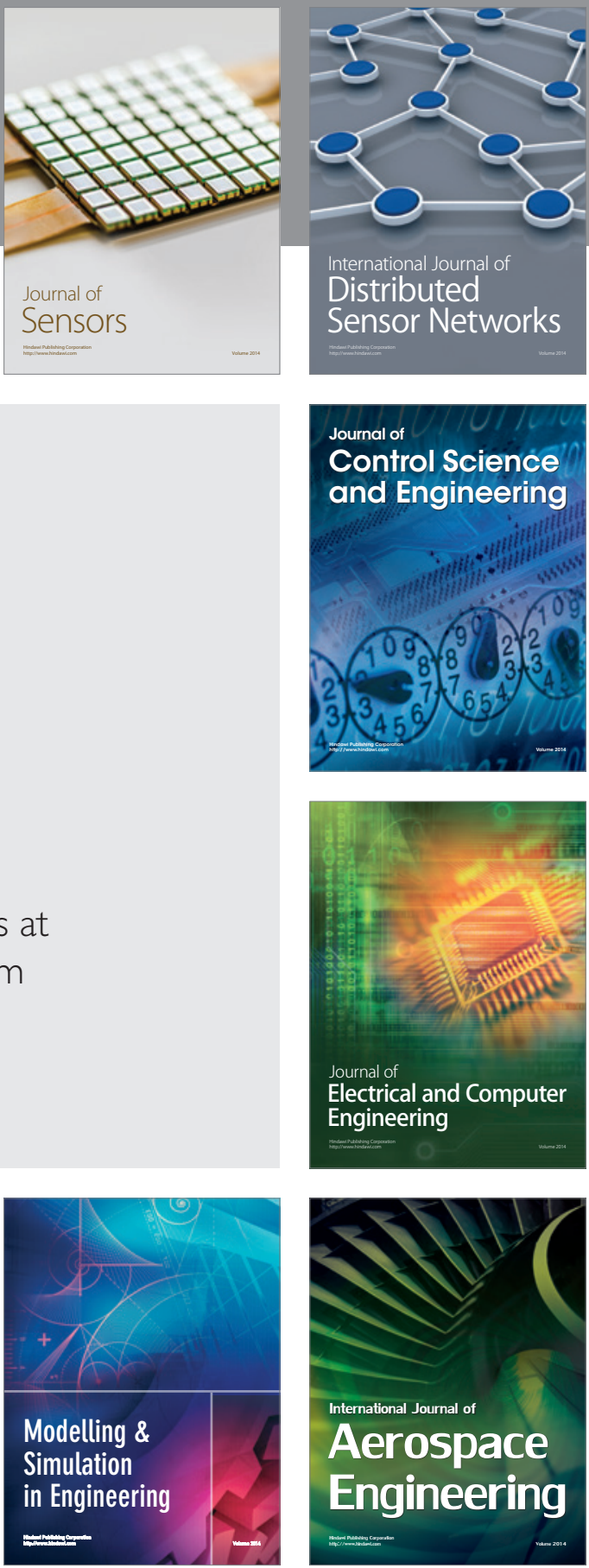

Journal of

Control Science

and Engineering
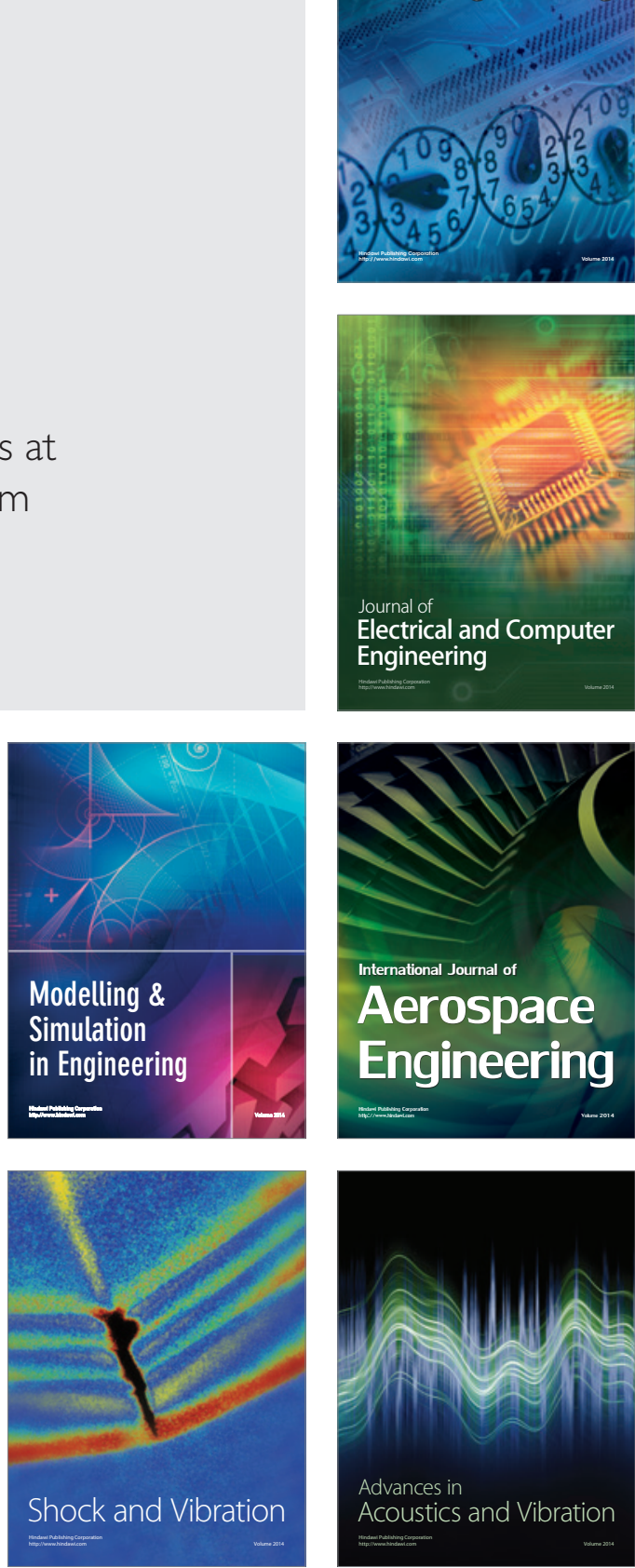